\title{
QRS Complex Analysis Using Wavelet Transform
}

\author{
Shobhana Yadav and A. K. Wadhwani \\ Electrical Engineering department \\ MITS, Gwalior, India \\ shobhanay@gmail.com,Wadhwani_arun@rediffmail.com
}

\begin{abstract}
The paper explains the method of detection of QRS complex from ECG signal using wavelet transform.By using MATLABtool, we can detect QRS complex which further helps us in diagnosis of various disease i.e. related to heart, when QRS complexes were detected then each complex was used to find the peaks of the waves like $Q, R \& S$.
\end{abstract}

\section{Introduction}

The heart is the main organ in the circulatory system. It pumps blood through blood vessels, from heart, goes out through the body and again ends back at the heart, the one cycle it complete is called cardiac cycle which occurs about 72 times per minute. There are two phases of the cardiac cycle one is diastole and other is systole. In the diastole phase, the heart ventricles are relaxed and the heart fills with blood .In the systole phase, the ventricles contract and pump blood to the arteries.

Electrocardiography is the electrical representation of the activity of the heart over a period of time. ECG is detected by using electrodes attached to the surface of body. The graph obtained is called electrocardiograph. The electrocardiogram is a graphical recording of the time variant voltages.

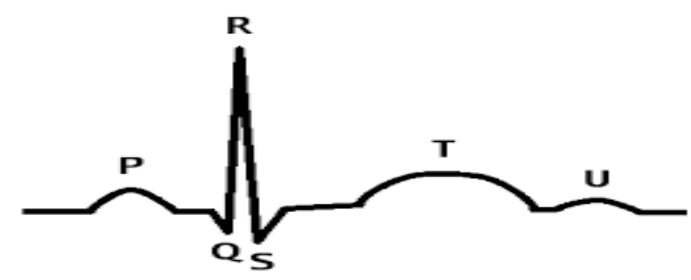

Figure 1. ECC Signal

At rest, each heart muscle cell is in polarized condition (i.e., inner surface of cell is negatively charged).Entry of $\mathrm{Na}+$ and $\mathrm{Ca}+$ make it positive called depolarization, which activate the cell and forced it to contract. The ECG signal is characterized by five peaks labeled by letters $\mathrm{P}, \mathrm{Q}, \mathrm{R}, \mathrm{S}, \mathrm{T}, \& \mathrm{U}($ in some cases). The $\mathrm{P}$ wave represents the depolarization of the atria while QRS represents the depolarization of ventricles. The detection of QRS complex is the most important task in ECG signal analysis.

Different Waves with their amplitude

Table 1. The ECG Wave Amplitudes

\begin{tabular}{|l|l|}
\hline Wave & Amplitude \\
\hline $\mathrm{P}$ wave & $0.25 \mathrm{mv}$ \\
\hline $\mathrm{R}$ wave & $1.60 \mathrm{mv}$ \\
\hline $\mathrm{Q}$ wave & $25 \%$ of $R$ wave \\
\hline T wave & 0.1 to $0.5 \mathrm{mv}$ \\
\hline
\end{tabular}


Different Waves with time durations

Table 2. The ECG Wave Duration

\begin{tabular}{|l|l|}
\hline Interval & Duration \\
\hline PR & 0.12 to 0.20 seconds \\
\hline PQ & Delay \\
\hline QRS & 0.06 to 0.1 seconds \\
\hline QT & 0.2 to 0.4 seconds \\
\hline RR & 0.6 to 0.12 seconds \\
\hline ST & 0.05 to 0.15 seconds \\
\hline
\end{tabular}

The P-, QRS- and T-waves reflect the rhythmic electrical depolarization and repolarization of the myocardium associated with the contractions of the atria and ventricles.

Electrical impulse (wave of depolarisation) is picked up by placing electrodes on patient body. The voltage change is sensed by measuring the current change across 2 electrodes - a positive electrode and a negative electrode. $f$ If the electrical impulse travels towards the positive electrode this result in a positive deflection. $f$ If the impulse travels away from the positive electrode this results in a negative deflection. Latest Research has showed that about 20 percent of all deaths occurred in India is because of heart problem. Maximum Population has been suffered from heart disease \& major reasons for cardiac arrest are increasing age, changing life style, taking high calorie food etc. To eliminate the chance of avoiding cardiac disease is becoming important day by day. For this we have to keep an eye over our health problem related to heart i.e. a continuous checkups is necessary to avoid any heart problem. In this paper we are presenting techniques to find out QRS complex which helps us in further diagnosis of disease.

\section{Wavelet Transform}

\section{Fourier Transforms}

Most of the raw signal is a function of time. This representation is not always the best representation of the signal for most signal processing related application. In many cases, information remains hidden in the frequency content of that signal. In short Fourier transform is the temporal representation of the signal. To overcome that problem wavelet transform is used.

\section{Wavelet}

Wavelet is a term used for small wave. It is used to analyze signal with short duration finite energy function. Wavelet analysis is the process of breaking a signal into shifted and scaled version of the original wavelet (mother wavelet). Wavelet functions deals in both time and frequency domain. This transform is suitable for non stationary signal. ECG signal has high frequency component like QRS complex. It can be shown that we can both have frequency and temporal information by this kind of transform using wavelets. 


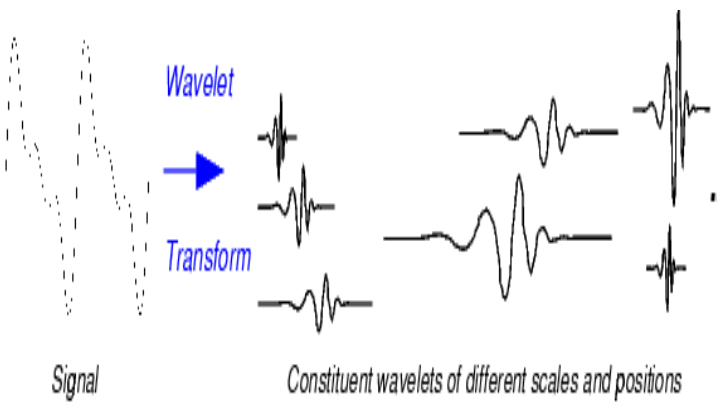

Wavelet transform used in many applications:

- Data and image compression

- Elimination of noise

- Signal compression

- Image compression

- Deriving transient signal features

Different wavelet families

- Daubechies

- Haar

- Symlets

- Coiflets

If a signal is passed through low pass and high pass filter then the signal is decomposed into two components. A detailed part-high frequency and approximation part-low frequency. The sub signal produced from low filter will have highest frequency equal to half of original frequency. Wavelet analysis make us using long time intervals when we need more precise low-frequency information, and shorter regions when we want highfrequency information. Fourier analysis consists of breaking up a signal into sine waves of various frequencies. Similarly, wavelet analysis is the breaking up of a signal into shifted and scaled versions of the original (or mother) wavelet.

\section{Methodology}

ECG data has been taken from physionet online. DB10 Wavelet is applied over original signal. On applying Db10 wavelet and decompose it till fourth stage will give us denoised, and important feature extraction. Soft Thresholding is applied to make signal less than 1 count as zero and signal more than 1 count as 1 .

After achieving QRS complex from the original signal, no. of beats is calculated. FP (false positive pulse) \& FN (false negative pulse) is determined. FP will determine validity of algorithm and FN will determine sensitivity.

$$
\text { Accuracy } \%=[1-(\mathrm{FP}+\mathrm{FN}) / \mathrm{Tp}] * 100
$$




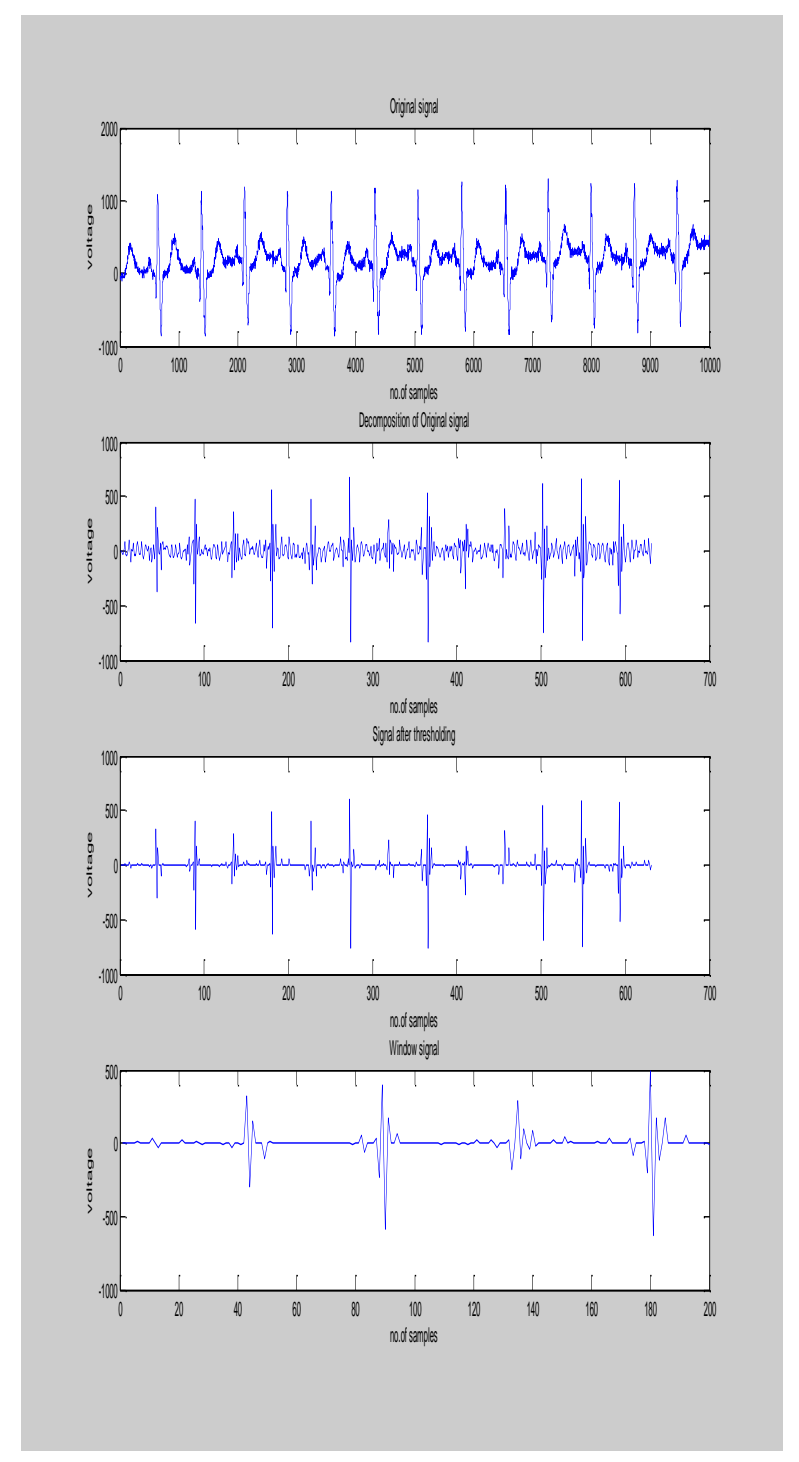

Figure 3. QRS Wave Detection

\section{Result}

Result we are getting from this algorithm is applied over PTB database, i.e., shown in Table no. 3 .

Table 3. QRS Wave Detection

\begin{tabular}{|l|l|l|l|l|}
\hline S.NO & $\begin{array}{l}\text { CONDITIO } \\
\text { N OF } \\
\text { HEART }\end{array}$ & $\begin{array}{l}\text { TOTA } \\
\text { L NO. } \\
\text { OF } \\
\text { BEAT } \\
\text { S }\end{array}$ & $\begin{array}{l}\text { FP+F } \\
\text { N }\end{array}$ & $\begin{array}{l}\text { ACCURAC } \\
\text { Y\% }\end{array}$ \\
\hline 01 & Arrhythmia & 11 & 0 & 100 \\
\hline 02 & $\begin{array}{c}\text { PTB } \\
\text { diagnostic }\end{array}$ & 9 & 0 & 100 \\
\hline 03 & MIMIC II & 13 & 0 & 100 \\
\hline 04 & stressed & 9 & 1 & 99.99 \\
\hline 05 & $\begin{array}{c}\text { Healthy } \\
\text { control }\end{array}$ & 10 & 0 & 100 \\
\hline
\end{tabular}




\section{Conclusion}

In my paper I have detected every parameters related to QRS complex in ECG signal which further help in disease diagnosis. In some test accuracy achieved is $100 \%$ which proves the validity of algorithm.

\section{Future Work}

We have used Daubechies family in QRS detection. Various wavelets can be used to improve the accuracy and sensitivity.

\section{References}

[1] H. Nagendra, "Application of wavelet Techniques in ECG signal processing: An overview", International journal of engineering science and Technology, vol. 3, no. 10, (2011) October, pp. 74327443.

[2] K. V. L. Narayana and A. B. Rao, "Wavelet based QRS detection in ECG using MATLAB", Innovation Systems Design and Engineering, vol. 2, no. 7, (2011).

[3] "Transform of the ECG Signal", The 2nd International Conference on Multimedia and Ubiquitous Engineering (MUE2008), Busan, Korea, (2008) April, pp. 201-206.

[4] G. Jaswal, R. Parmar and A. Kaul, "QRS Detection Using Wavelet Transform”, International Journal of Engineering and Advanced Technology (IJEAT), vol. 1, (2012) August.

[5] C. Li, C. Zheng and C. Tai, "Detection of ECG characteristic points using Wavelet Transforms", vol. 42, no. 1 , (1995) January, pp. 21-28.

[6] S. Kadambe, R. Murray and G. F. Boudreaux-Bartels, "Wavelet transform based QRS complex detector", vol. 46, no. 7, (1999) July, pp. 838-848.

[7] C. Li, C. Zheng and C. Tai, "Detection of ECG Characteristic points using Wavelet Transforms", IEEE Trans. Biomed. Eng., vol. 42, (1995), pp. 1.

[8] S. Karpagachelvi, M. Arthanari and M. Sivakumar, "QRS Wave Detection Using Multi resolution Analysis", vol. 10, (2010), pp. 39-42. 
International Journal of Bio-Science and Bio-Technology

Vol.7, No.6 (2015) 